Apidologie, 1981, $12(4), 345-362$.

\title{
POLLINISATION DU COLZA D'HIVER MALE-FERTILE ET MALE-STÉRILE (Brassica napus L. var. oleifera METZGER) PAR L'ABEILLE DOMESTIQUE (Apis m. mellifica L.). EFFETS SUR LA PHÉNOLOGIE ET LE RENDEMENT
}

\author{
J. MESQUIDA \\ I.N.R.A., Laboratoire de Zoologie, Domaine de la Motte-au-Vicomte, B.P. 29, 35650 Le Rheu. \\ M. RENARD \\ I.N.R.A., Station d'Amélioration des Plantes, Domaine de la Motte-au-Vicomte, \\ B.P. 29, 35650 Le Rheu
}

\begin{abstract}
RÉSUMÉ
Les auteurs ont étudié l'effet de la pollinisation sur quelques caractères phénologiques et sur quelques composantes du rendement du colza d'hiver mâle-fertile (variété "Primor ») et mâle-stérile d'origine cytoplasmique.

L'effet du butinage s'est surtout manifesté, sur les plantes mâle-stériles seulement, par une augmentation significative de certains caractères (nombre de fleurs, nouaisons, nombre de siliques, nombre de graines par silique, production et rendement en graines) ainsi que par une diminution significative d'autres caractères (durée de floraison, taille des ramifications primaires, production de fleurs et taille des graines).

Ces mêmes effets sont également apparus sur les plantes mâle-fertiles mais plus atténués et non significatifs, sauf en ce qui concerne les nouaisons et la production grainière de 1000 fleurs. Le rendement en graines des plantes mâle-fertiles n'a finalement augmenté que de $6 \%$.

L'effet dû au vent seul a été non négligeable sur les nouaisons des plantes mâle-stériles mais les rendements ont été au contraire négligeables.
\end{abstract}

\section{INTRODUCTION}

La pollinisation est un processus complexe qui semble agir de manière différente sur de nombreuses parties de la plante, et plus particulièrement sur la formation des fruits et la production grainière. Mais le rôle joué par les insectes pollinisateurs sur le développement de la plante et sur la production grainière du colza (Brassica napus $\mathrm{L}$. 
var. oleifera METZGER) n'apparaît pas clairement et suscite de nombreuses divergences d'opinion chez les auteurs.

Ainsi, les auteurs s'accordent en général à reconnaitre que la durée de floraison peut être plus courte lorsqu'il y a pollinisation par les insectes (EWERT, 1929; ZANDER, 1952). BARBIER (1978) précise que les fleurs de colza visitées par les abeilles ont une durée de vie de trois jours contre cinq jours pour celles qui ne le sont pas. En l'absence d'abeilles, Williams (1978), de son côté, ne trouve pas de différences significatives lorsqu'il compare l'autopollinisation et la pollinisation croisée manuelle.

La pollinisation par les abeilles augmente les taux de nouaison (EIsıKowITCH, 1981 ) et les ramifications sont moins longues (BARBIER, 1977-1978); les siliques sont plus longues (EWERT, 1929; MEYERHOFF, 1954; BARBIER, 1978) et plus nombreuses par pied (MEYERHOFF, 1954 et KOUTENSKy, 1959); elles se forment plus tôt (EwERT, 1929), mûrissent plus tôt (WILLIAMS, 1980) et renferment davantage de graines (MEYERHOFF, 1954; KOUTENSKY, 1959; ForSTER et al., 1973; BARBIER, 1978; EISIKOWITCH, 1981) ou non selon Van PrAAGH et al. (1979); les graines sont plus grosses (MEYERHOFF, 1954) ou plus petites (BARBIER, 1978; FrEE et NuTTALL, 1968) et parfois ont une teneur en huile un peu élevée (Van PraAgh et al. 1979). Dans ces divers aspects, il semblerait cependant que les insectes ne soient pas forcément en cause, puisque WILLIAMS (1978) obtient plus de siliques et plus de graines par silique par simple secouement des plantes. D'ailleurs, de nombreux auteurs considèrent qu'en raison de son autofertilité caractéristique, le colza produit suffisamment de graines sans l'intervention des insectes pollinisateurs (Nicolaisen, 1943; HARle, 1951; BeCker, 1951; Persson, 1956; Williams, 1976; Van Praagh et al., 1979). Williams (1978) ne trouve d'ailleurs pas de différences de rendement entre la pollinisation manuelle en serre et la pollinisation au champ avec les pollinisateurs. Cependant, certains auteurs constatent, avec butinage, de légers accroissements du rendement en graines qu'ils considèrent comme non négligeable, même s'ils n'observent pas de différences significatives (FuJITA, 1939; ZANDER, 1052; Rhein, 1952; Jenkinson et GlynNe-Jones, 1953; PritsCh, 1965 et Free et Nuttall, 1968 pour des essais sous cages, et Meyerhoff, 1954; Koutens-: KY, 1959, sur colza Brassica napus var. arvensis; Belozerova, 1960; DEMIANOWICZ, 1965 , pour des observations de plein champ.

Ajoutons que l'autopollinisation du colza, qui semble être différente selon les variétés (Williams, 1978), est cependant responsable de 30 à $50 \%$ des rendements selon HARLe (1948) et JENKINSON et GlyNNE-Jones (1953), en conditions de serre, et de $70 \%$ environ dans des conditions de culture, d'après Olsson (1952 et 1955), Rives (1957), Downey et al. (1970), White (1970) et MORICE (1976). La fécondation croisée augmenterait les rendements de $14 \%$ selon FECHNER (1927) alors que NicolaiseN (1943) et Williams (1978) ne trouvent aucune différence. Il faut savoir également que le rendement du colza résulte aussi de l'influence de facteurs du milieu (climat et fertilité du sol), des potentialités génétiques de la plante (RolliER, 1974) et des phénomènes de compensation. 
Notons aussi qu'il existe des corrélations entre les différents caractères végétatifs et les facteurs de rendement du colza mis en évidence par BAUR (1943), SCHRIMPF (1954), Stolle (1954), Rives (1957), et Morice (1960).

En conséquence, malgré les nombreuses incertitudes relatives aux bénéfices que peut tirer le colza de la visite des fleurs par les abeilles, on peut penser qu'une mauvaise pollinisation peut parfois limiter les rendements.

Les études poursuivies à Rennes, et orientées sur la production de semences hybrides $\mathrm{F} 1$ de colza avec utilisation de la stérilité mâle cytoplasmique, nous ont amené à nous intéresser à l'effet de la pollinisation par les abeilles sur deux types de colza : la variété "Primor " à fleurs hermaphrodites, et une lignée mâle-stérile. Nous avons étudié les répercussions de la visite des insectes sur le développement des plantes et leur production grainière en nous limitant à quelques aspects de la phénologie et aux principales composantes du rendement. Pour cela, nous avons effectué, pendant deux années consécutives (1977 et 1978) des études comparatives sous cages avec et sans abeilles, et des études de plein champ.

\section{MATÉRIEL ET MÉTHODES}

A. - Le matériel végétal

On a utilisé la variété " Primor " comme colza mâle-fertile en raison de son homogénéité et de sa participation à la constitution génétique du matériel végétal mâle-stérile utilisé.

Le colza mâle-stérile était la lignée $\mathrm{n}^{\circ}$ 3-22-3-7. Il s'agit d'une stérilité mâle cytoplasmique provenant d'un croisement entre un radis mâle-stérile (Raphanus sativus L.) et un chou (Brassica oleracea L.), puis avec un colza fertile (variété « Primor $»)$. Cette stérilité mâle a fait l'objet d'études sur la pollinisation en vue de la production de semences hybrides F 1 (Mesquida et Renard, 1978 et 1979, Renard et Rous SELLe, non publié, Rousselle, 1981). Il s'agit d'une stérilité mâle complète et stable.

B. - Les essais

On a effectué au Rheu (Ille-et-Vilaine) en 1977 et en 1978 deux essais (essai 1 et essai II).

L'essai I (1977) était situé à une centaine de mètres d'un rucher de 15 ruches et en bordure nord d'un champ de colza d'hiver d'une superficie d'un hectare environ. Il etait abrité des vents du nord par un bois. C'était un essai bloc à trois répétitions comprenant trois traitements :

1. Parcelles sous cages avec abeilles.

2. Parcelles sous cages sans abeilles.

3. Parcelles de plein champ sans cages.

L'essai II (1978) était à découvert, au milieu d'un champ de colza plus étendu ( $3 \mathrm{ha}$ ) à environ $500 \mathrm{~m}$ du rucher. C'était un essai bloc randomisé à six répétitions et comprenant les mêmes traitements que ceux de l'essai I.

Dans chaque parcelle des deux essais, on a disposé une bande de trois lignes de $3 \mathrm{~m}$ de long et $0,50 \mathrm{~m}$ d'intervalle de colza d'hiver mâle-fertile et une bande de trois lignes de mêmes dimensions de colza d'hiver mâle-stérile. Les colzas ont été semés en septembre à raison de $5 \mathrm{~kg}$ de graines à l'hectare. L'écartement entre parcelles était d'un mètre pour l'essai I et de trois mètres pour l'essai II.

Du début de la floraison jusqu'à la récolte du colza, on a mis en place des cages de $18 \mathrm{~m}^{3}$ $(3 \times 3 \times 2 \mathrm{~m})$, recouvertes d'une toile plastique à mailles de $2 \mathrm{~mm}$, qui interdit tout accès des insectes polli- 
nisateurs sauvages de l'extérieur. Ce type de toile diminue la vitesse du vent de $90 \%$ pour un vent de $2750 \mathrm{~m} / \mathrm{h}$ et de $60 \%$ pour un vent de $4500 \mathrm{~m} / \mathrm{h}$.

La pollinisation dans les cages sans abeilles était assurée par le vent seul. Dans les parcelles de plein champ, on a laissé agir normalement les agents pollinisateurs locaux (vent + abeilles).

Dans chaque cage avec abeilles, on a introduit pour toute la durée des floraisons des colzas mâlefertiles et mâle-stériles, une ruchette à deux cadres " Dadant " peuplée d'abeilles sans reine et renfermant au moins un cadre de couvain ouvert. On a maintenu en permanence, à l'intérieur des cages et dans des coupelles, du sirop de sucre concentré et de l'eau.

\section{C. - Observations effectuées}

Les observations ont porté essentiellement sur le comportement de butinage, sur des caractères phénologiques et sur les principales composantes du rendement des deux types de colza, à savoir :

- nombre de butineuses présentes sur les plantes mâle-fertiles et mâle-stériles des cages et des parcel. les de plein champ à différents moments de la période de floraison du colza;

- évolution des floraisons par estimation du nombre de fleurs butinables au mètre carré faite à diffé. rentes périodes selon la méthode de comptage du cerceau de $50 \mathrm{~cm}$ de diamètre préconisée par DeLAUDE e1 TASEI (1972);

- taux de nouaison (nombre de siliques formées/nombre total de fleurs $\times 100$ ) estimés sur les 50 premières fleurs de la ramification primaire de 5 pieds mâle-fertiles et 5 pieds mâle-stériles tirés au hasard dans chaque parcelle.

On a également évalué, après maturation, le développement des plantes en prenant dans chaque parcelle, au hasard, 5 pieds mâle-fertiles et 5 pieds mâle-stériles dont on a mesuré :

- la hauteur totale du collet à l'extrémité de la ramification primaire;

- la longueur de la ramification primaire;

- le nombre total de fleurs sur la ramification primaire.

Les composantes du rendement qui ont retenu notre attention étaient :

- le nombre de siliques formées;

- le nombre de graines par silique;

- le poids de 1000 graines.

Cela nous a permis de calculer la production grainière ( $R$ 1) pour 1000 fleurs butinables au mètre carré. Ces composantes du rendement ont été évaluées sur les ramifications primaires des 5 pieds mâlefertiles et 5 pieds mâle-stériles tirés au hasard dans chaque parcelle.

Enfin, on a mesuré le rendement en graines des plantes entières ( $R$ 2). Les poids des graines et les rendements ont été donnés en poids secs.

Les comparaisons de moyennes ont été traitées au moyen de l'analyse de la variance après vérification des conditions d'emploi et après transformation de données en logarithmes pour les numérations brutes et transformation angulaire de BLISs ( $\operatorname{arc} \sin \sqrt{ } \mathrm{P}$ ) pour les pourcentages (taux de nouaison).

\section{RÉSULTATS}

\section{Comportement de butinage des abeilles}

D'une manière générale, en cage et à l'extérieur, les fleurs des plantes mâle-fertiles ont toujours été significativement plus attractives que les fleurs des plantes mâle-stériles (tableau 1). Mais l'activité de butinage a été beaucoup plus intense dans les cages qu'en extérieur. 
TABL. 1. - Nombres moyens d'abeilles butineuses par cage $\left(3 \mathrm{~m}^{2}\right)$

à différentes dates sur fleurs de colza mâle-fertile

et mâle-stérile dans les cages avec abeilles $(+A b)$ et de plein champ (T) : essai I, 1977.

NS : différence non significative

$\mathrm{S}^{*}$ : moyennes significativement différentes à $\mathrm{P}=5 \%$

$\mathrm{S}^{* *}$ : moyennes significativement différentes à $\mathrm{P}=1 \%$

$\mathrm{S}^{* * *}$ : moyennes hautement significatives à $\mathrm{P}=1 \%$.

$( \pm): \pm$ erreur type $: \pm s / \sqrt{ } n$ ( $s=$ écart type)

TABL. 1. - Mean numbers of foraging honeybees per cage $\left(3 \mathrm{~m}^{2}\right)$ at different dates on flowers of male-fertile and male-sterile rape seed

in cages with honeybees (+ Hbees) and in the field (T) : test I in 1977.

NS : difference not significant

$\mathrm{S}^{*} \quad: \mathrm{P}=5 \%$ significant difference

$S^{* *}: P=1 \%$ significant difference

$\mathrm{S}^{* * * *}$ : highly significant averages

$( \pm) \quad: \pm$ standard error : $\pm \mathrm{S} / \sqrt{ } n(\mathrm{~S}=$ standard deviation $)$

\begin{tabular}{|c|c|c|c|c|c|}
\hline $\begin{array}{l}\text { Dates } \\
\text { Date }\end{array}$ & $\begin{array}{l}\text { Lignée } \\
\text { Strain }\end{array}$ & $\begin{array}{l}+\mathrm{Ab} \\
+ \text { Hbees }\end{array}$ & $\mathrm{T}$ & $\begin{array}{l}\text { Différences } \\
\text { Differences }\end{array}$ & $+\mathrm{Ab} / \mathrm{T}$ \\
\hline \multirow{2}{*}{$\begin{array}{c}27 / \mathrm{IV} \\
\left(17^{\mathrm{e}} \text { sem. }\right)\end{array}$} & $\begin{array}{l}\text { Mâle-fertile } \\
\text { Male-fertile }\end{array}$ & $\begin{array}{ll} & 6,0 \\
\pm & 1,73)\end{array}$ & $\begin{aligned} & 1,33 \\
( \pm & 0,33)\end{aligned}$ & N.S. & 4,51 \\
\hline & $\begin{array}{l}\text { Mâle-stérile } \\
\text { Male-sterile }\end{array}$ & $\begin{array}{c}2,0 \\
\pm \quad 1,15)\end{array}$ & $\begin{aligned} & 0,33 \\
( \pm & 0,33)\end{aligned}$ & N.S. & 6,06 \\
\hline \multicolumn{2}{|c|}{$\begin{array}{l}\text { Différences } \\
\text { Differences }\end{array}$} & N.S. & N.S. & & \\
\hline \multirow{2}{*}{$\begin{array}{c}3 / \mathrm{V} \\
\left(18^{\mathrm{e}} \mathrm{sem} .\right)\end{array}$} & $\begin{array}{l}\text { Mâle-fertile } \\
\text { Male-fertile }\end{array}$ & $\begin{array}{c}16,33 \\
\pm \quad 2,96)\end{array}$ & $\begin{array}{c}1,67 \\
\pm \quad 0,88)\end{array}$ & $\mathrm{S}^{* * *}$ & 9,78 \\
\hline & $\begin{array}{l}\text { Mâle-stérile } \\
\text { Male-sterile }\end{array}$ & $\begin{aligned} & 1,67 \\
& \pm \quad 1,20)\end{aligned}$ & $\begin{aligned} & 0,33 \\
( \pm \quad & 0,33)\end{aligned}$ & N.S. & 5,06 \\
\hline \multicolumn{2}{|c|}{$\begin{array}{l}\text { Différences } \\
\text { Differences }\end{array}$} & $\mathrm{S}^{*}$ & N.S. & & \\
\hline \multirow{2}{*}{$\begin{array}{c}13 / \mathrm{V} \\
\left(19^{\mathrm{e}} \text { sem. }\right)\end{array}$} & $\begin{array}{l}\text { Mâle-fertile } \\
\text { Male-fertile }\end{array}$ & $\begin{array}{r}\quad 8,33 \\
( \pm \quad 3,33)\end{array}$ & $\begin{array}{ll} & 5,33 \\
\pm \quad & 0,88)\end{array}$ & N.S. & 1.56 \\
\hline & $\begin{array}{l}\text { Mâle-stérile } \\
\text { Male-sterile }\end{array}$ & $\begin{array}{r}3,00 \\
( \pm \quad 1,53)\end{array}$ & $\begin{array}{c}2,0 \\
\pm \quad 0,58)\end{array}$ & N.S. & 1,5 \\
\hline \multicolumn{2}{|c|}{$\begin{array}{l}\text { Différences } \\
\text { Differences }\end{array}$} & N.S. & $\mathrm{S}^{*}$ & & \\
\hline \multirow{2}{*}{$\begin{array}{c}27 / \mathrm{V} \\
\left(21^{\mathrm{e}} \text { sem. }\right)\end{array}$} & $\begin{array}{l}\text { Mâle-fertile } \\
\text { Male-fertile }\end{array}$ & $\begin{array}{r}47,0 \\
+10,8)\end{array}$ & $\begin{array}{c}20,33 \\
+\quad 1,45)\end{array}$ & $\mathrm{S}^{*}$ & 2.31 \\
\hline & $\begin{array}{l}\text { Mâle-stérile } \\
\text { Male-sterile }\end{array}$ & $\begin{aligned} & 9,33 \\
&( \pm \quad 2,19)\end{aligned}$ & $\begin{aligned} & 4,33 \\
\pm \quad & 0,88)\end{aligned}$ & N.S. & 2,15 \\
\hline \multicolumn{2}{|c|}{$\begin{array}{l}\text { Différences } \\
\text { Differences }\end{array}$} & $\mathrm{S}^{*}$ & $S^{* *}$ & & \\
\hline
\end{tabular}

En effet, on trouve entre 1,6 et 9,8 fois plus d'abeilles dans les cages qu'en extérieur sur les fleurs mâle-fertiles et entre 1,5 et 6 fois plus sur les fleurs mâle-stériles (tableau 1). 
2. Densité des fleurs et durée des floraisons

Le tableau 2 montre qu'à partir de la troisième semaine de floraison (13 mai) dans les cages sans abeilles, il y a plus de fleurs que dans les cages avec abeilles (fig. $1 a$ et $b$ ).

La durée de la floraison est de 10 à 11 semaines environ sans abeilles et de 8 semaines avec abeilles (fig. $1 a$ et $b$ ).
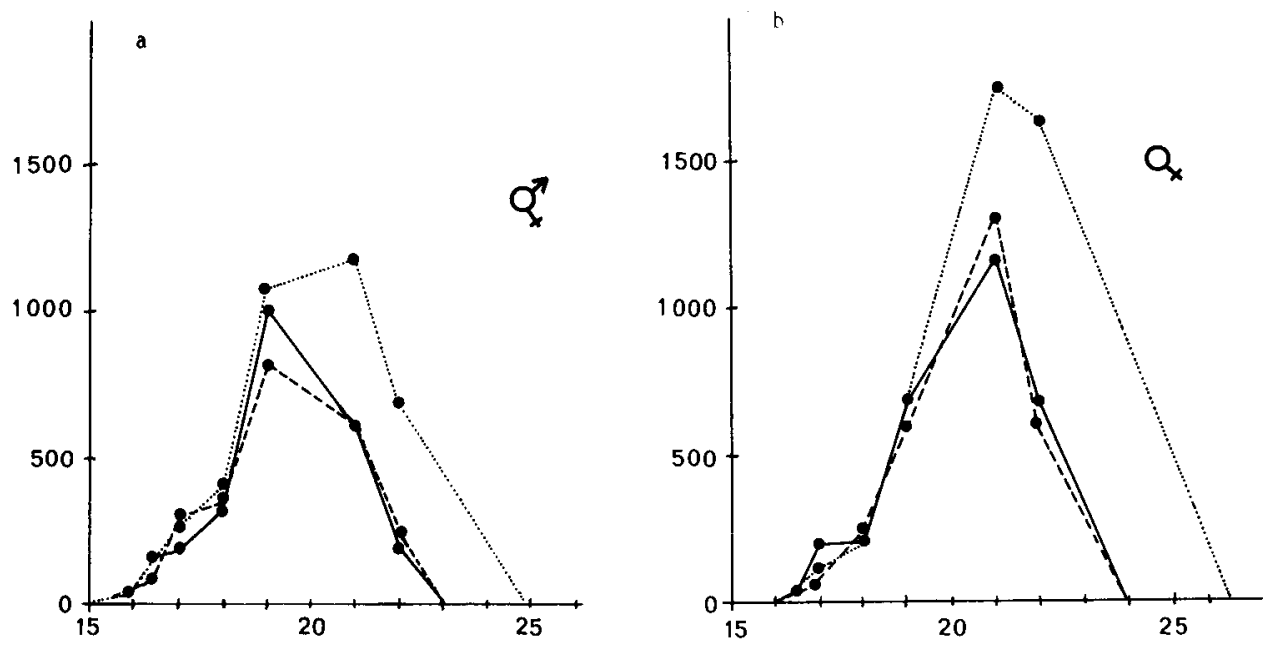

Fig. 1. - Courbes d'évolution moyenne des fleurs de colza mâle-fertile (l a) et mâle-stérile $(1 \mathrm{~b})$ dans les cages avec

en abscisse : les semaines

et sans abeilles et de plein champ de l'essai $I, 1977$.

en ordonnées : nombre de fleurs au mètre carré

: courbe moyenne de plein champ

-

: courbe moyenne des cages sans abeilles

$\checkmark \quad$ : colza mâle-fertile

: : colza mâle-stérile

FIG. 1. - Plot of mean evolution of the flowers of male-fertile (1 a) and male-sterile ( 1 b) winter rape-seed in cages with and without honeybees and in the field $(T)$ for the test I, in 1977.

$x$ axis : weeks

$y$ axis : number of flowers per square metre : mean curve in the field

mean curve of cages with honeybees

: meancurves of cages without honeybees

$q^{\circ}$ : male-fertile rape-seed

$\uparrow$ : male-sterile rape-seed

\section{Nouaisons}

Dans les cages avec abeilles, les moyennes ne sont pas significativement différentes de celles des parcelles de plein champ (tableau 3). Par contre, des différences hautement significatives apparaissent lorsqu'on compare ces moyennes à celles des cages sans abeilles. L'accroissement de nouaison chez les plantes mâle-fertiles avec abeilles 
TABL. 2. - Nombres moyens de fleurs de colza au mètre carré dans les cages avec $(+A b)$ et sans $(-A b)$ abeilles et de plein champ $(T)$ : essai $I, 1977$. Même légende que tableau 1.

Les moyennes suivies de la même lettre sont significativement différentes.

TABL. 2. - Mean numbers of rape-seed flowers per square metre in cages with (+ Hbees) and without (- Hbees) honeybees and in the field (T): test I in 1977.

Legend as in Table 1.

Averages followed by the same letter are significantly different.

\begin{tabular}{|c|c|c|c|c|c|c|c|}
\hline \multirow{2}{*}{$\begin{array}{l}\text { Dates } \\
\text { Dates }\end{array}$} & \multicolumn{2}{|c|}{$\begin{array}{l}\text { Lignée mâle-fertile } \\
\text { Male-fertile strain }\end{array}$} & & \multicolumn{3}{|c|}{$\begin{array}{l}\text { Lignée mâle-stérile } \\
\text { Male sterile strain }\end{array}$} & \\
\hline & $\begin{array}{l}+\mathrm{Ab} \\
+ \text { Hbees }\end{array}$ & $\begin{array}{c}-\mathrm{Ab} \\
- \text { Hbees }\end{array}$ & & $\begin{aligned} & +\mathrm{Ab} \\
+ & \text { Hbees }\end{aligned}$ & $\begin{array}{c}-\mathrm{Ab} \\
- \text { Hbees }\end{array}$ & $T$ & \\
\hline $\begin{array}{c}19 / \mathrm{IV} \\
\left(16^{\mathrm{c}} \text { sem. }\right)\end{array}$ & $\begin{array}{r}48,33 \\
( \pm \quad 6,96)( \pm\end{array}$ & $\begin{array}{c}39,67 \quad 44,0 \\
4,49)( \pm \quad 4,62)\end{array}$ & N.S. & $\begin{array}{l}2,3 \\
0,3)\end{array}$ & $\begin{array}{l}1,4 \\
0,6) \quad( \pm\end{array}$ & $\begin{array}{l}3,0 \\
0,6)\end{array}$ & N.S. \\
\hline $\begin{array}{c}22 / \mathrm{IV} \\
\left(16^{\mathrm{e}} \mathrm{sem} .\right)\end{array}$ & $\begin{array}{r}101,7 \\
( \pm 23,1)(t\end{array}$ & $\begin{array}{r}128,3 \\
+\quad 43,4)\left(\begin{array}{r}150,0 \\
40,7\end{array}\right)\end{array}$ & N.S. & $\begin{array}{l}28,3 \\
10,9)\end{array}$ & $\begin{array}{c}20,0 \\
2,9) \quad( \pm\end{array}$ & $\begin{array}{r}26,7 \\
4,4)\end{array}$ & N.S. \\
\hline $\begin{array}{c}27 / \mathrm{IV} \\
\left(17^{\mathrm{e}} \text { sem. }\right)\end{array}$ & $\begin{array}{r}291,7 \\
( \pm \quad 29,5)( \pm\end{array}$ & $\left.\begin{array}{r}253,3 \\
\pm \quad 60,9)( \pm \quad 8,7\end{array}\right)$ & N.S. & $\begin{array}{l}63,3 \\
18,6)\end{array}$ & $\begin{array}{c}110,0 \\
27,5) \quad( \pm\end{array}$ & $\begin{array}{r}195,0 \\
46,5)\end{array}$ & N.S. \\
\hline $\begin{array}{c}3 / \mathrm{V} \\
\left(18^{\mathrm{e}} \text { sem. }\right)\end{array}$ & $\begin{aligned} & 350,0 \\
&( \pm 135,9)\end{aligned}$ & $\begin{array}{r}396,7 \\
+\quad 86,6)( \pm 115,7\end{array}$ & N.S. & $\begin{array}{r}235,0 \\
51,1)\end{array}$ & $\begin{array}{c}198,3 \\
30,6) \quad( \pm\end{array}$ & $\begin{array}{r}210,0 \\
27,8)\end{array}$ & N.S. \\
\hline $\begin{array}{c}13 / V \\
\left(19^{e} \text { sem. }\right)\end{array}$ & $\begin{array}{r}815,0 \\
( \pm 154,6)(t\end{array}$ & $\begin{array}{rc}1083,3 & 997,5 \\
\pm \quad 136,4)( \pm 217,5)\end{array}$ & N.S. & $\begin{array}{r}581,7 \\
\pm \quad 73,7)\end{array}$ & $\begin{array}{r}686,7 \\
( \pm \quad 66,4)( \pm\end{array}$ & $\begin{array}{l}677,5 \\
297,5)\end{array}$ & N.S. \\
\hline $\begin{array}{c}27 / V \\
\left(21^{\mathrm{e}} \text { sem. }\right)\end{array}$ & $\left(\begin{array}{c}611,7 \\
24,9 \\
(a)\end{array}\right)(t$ & 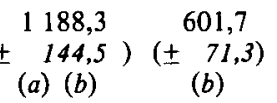 & $S^{* *}$ & $\begin{array}{c}1325,0 \\
\pm \quad 100,5) \\
(a)\end{array}$ & $\begin{array}{c}1771,7 \\
\pm \quad 68,7) \\
(a)(b)\end{array}$ & $\begin{array}{l}170,0 \\
115,3) \\
(b)\end{array}$ & $\mathrm{S}^{* *}$ \\
\hline $\begin{array}{c}3 / \mathrm{VI} \\
\left(22^{e} \text { sem. }\right)\end{array}$ & $\begin{array}{r}238,3 \\
( \pm .40,6)(t \\
(a)\end{array}$ & $\begin{array}{c}708,3 \\
\left.+\quad \begin{array}{c}203,3 \\
79,5\end{array}\right)\left( \pm \begin{array}{c}28,9 \\
(b)\end{array}\right)\end{array}$ & $\mathrm{S}^{* * *}$ & $\left(\begin{array}{l}606,7 \\
\pm \quad 171,8) \\
(a)\end{array}\right.$ & $\begin{array}{c}1658,3 \\
\left(\begin{array}{c}258,7) \\
(a)(b)\end{array}\right.\end{array}$ & $\begin{array}{l}678,3 \\
118,9) \\
(b)\end{array}$ & $S^{* *}$ \\
\hline
\end{tabular}

est de $18 \%$ dans l'essai II et $39 \%$ dans l'essai I. Dans le cas des plantes mâle-stériles, cette augmentation est plus élevée : $89 \%$ dans l'essai II et $500 \%$ dans l'essai I. Il faut souligner que les taux de nouaisons de $14,1 \%( \pm 2,4)$ et $42,8 \%( \pm 1,2)$ obtenus sur les plantes mâle-stériles non butinées sont dus strictement à la pollinisation anémophile.

\section{Hauteur des plantes et longueur des ramifications primaires}

On a mis en évidence un " effet cage " et un " effet pollinisation " sur le développement des plantes mâle-fertiles et mâle-stériles des cages, ainsi que sur les longueurs moyennes de leurs ramifications primaires toujours significativement plus élevées que celles des plantes de plein champ (tableau 4).

Par ailleurs, les plantes et les ramifications primaires sont toujours plus longues dans les cages sans abeilles. Cela est plus net chez les plantes mâle-stériles que chez les plantes mâle-fertiles. 
TABL. 3. - Taux moyens de nouaison des ramifications primaires des plantes de colza mâle-fertile et mâle-stérile dans les cages

avec $(+A b)$ et sans $(-A b)$ abeilles et de plein champ $(T)$ : essais I, 1977 et II, 1978.

TABL. 3. - Mean rates of fruit set of the primary ramifications

of male-fertile and male-sterile rape seed plants in cages with (+ Hbees)

and without (-Hbees) honeybees and in the field $(T):$ Test 1 , in 1977 and II, in 1978 .

\begin{tabular}{|c|c|c|c|c|c|c|c|c|}
\hline & & $\begin{array}{l}+\mathrm{Ab} \\
+ \text { Hbees }\end{array}$ & $\begin{array}{l}-A b \\
- \text { Hbees }\end{array}$ & $\mathrm{T}$ & & $\begin{array}{c}+\mathrm{Ab} \\
/ \mathrm{T}\end{array}$ & $\begin{array}{c}-\mathrm{Ab} \\
/ \mathrm{T}\end{array}$ & $\begin{array}{l}+A b \\
1-A b\end{array}$ \\
\hline $\begin{array}{c}\text { Essai } \\
\text { I }\end{array}$ & $\begin{array}{l}\text { Mâle-fertile } \\
\text { Male-fertile }\end{array}$ & $\left(\begin{array}{c}91,73 \\
\pm \quad 2,46) \\
(a)\end{array}\right.$ & $\begin{array}{c}65,6 \\
\left(\begin{array}{c}4,87) \\
(a)(b)\end{array}\right.\end{array}$ & $\left(\begin{array}{c}88,8 \\
\pm \quad 4,10) \\
(b)\end{array}\right.$ & $S^{* * *}$ & 1,03 & 0,74 & 1,39 \\
\hline $\begin{array}{c}\text { Test } \\
\text { I }\end{array}$ & $\begin{array}{l}\text { Mâle-stérile } \\
\text { Male-sterile }\end{array}$ & $\left(\begin{array}{c}84,8 \\
\pm \begin{array}{c}2,39) \\
(a)\end{array}\end{array}\right.$ & $\begin{array}{c}14,13 \\
( \pm \quad 2,36) \\
(a)(b)\end{array}$ & $\begin{array}{c}76,8 \\
\pm \begin{array}{c}4,66) \\
(b)\end{array}\end{array}$ & $\mathrm{S}^{* * *}$ & 1,10 & 0,18 & 6,00 \\
\hline \multicolumn{2}{|c|}{$\begin{array}{l}\text { Différences } \\
\text { Differences }\end{array}$} & N.S. & $\mathrm{S}^{* * *}$ & $\mathrm{~S}^{* * *}$ & & & & \\
\hline $\begin{array}{c}\text { Essai } \\
\text { II }\end{array}$ & $\begin{array}{l}\text { Mâle-fertile } \\
\text { Male-fertile }\end{array}$ & $\begin{array}{c}89,0 \\
\pm \quad 2,14) \\
(a)\end{array}$ & $\begin{array}{c}75,5 \\
\pm \quad 4,19) \\
(a)(b)\end{array}$ & $\begin{array}{c}93,23 \\
\pm \quad 1,53) \\
(b)\end{array}$ & $\mathrm{S}^{* *}$ & 0,95 & 0,81 & 1,18 \\
\hline $\begin{array}{c}\text { Test } \\
\text { II }\end{array}$ & $\begin{array}{l}\text { Mâle-stérile } \\
\text { Male-sterile }\end{array}$ & $\left(\begin{array}{c}80,87 \\
+\begin{array}{c}2,98) \\
(a)\end{array}\end{array}\right.$ & $\begin{array}{c}42,8 \\
\left(\begin{array}{c}1,22) \\
(a)\end{array}\right)\end{array}$ & $\left(\begin{array}{c}84,47 \\
\pm(b) \\
(b)\end{array}\right.$ & $S^{* * *}$ & 0,96 & 0,51 & 1,89 \\
\hline & $\begin{array}{l}\text { érences } \\
\text { erences }\end{array}$ & $\mathrm{S}^{* * *}$ & $\mathrm{~S}^{* * *}$ & $S^{* * *}$ & & & & \\
\hline
\end{tabular}

Même légende que tableaux 1 et 2.

Données en \%.

\section{Nombre de fleurs et nombre de siliques des ramifications primaires}

Trois résultats sont à retenir (tableau 5) :

- il n'y a pas de différences significatives de production de fleurs ou de siliques sur les ramifications primaires des plantes mâle-fertiles butinées ou non;

- les plantes mâle-stériles donnent significativement plus de fleurs que les plantes mâle-fertiles, que ce soit en cages avec ou sans abeilles ou en extérieur (15 à $33 \%$ de plus selon le traitement);

- en absence d'abeilles, les plantes mâle-stériles donnent moins de siliques. Par contre, en présence d'abeilles dans les cages, ces plantes donnent significativement plus de siliques que les plantes mâle-fertiles.

\section{Nombre de graines par silique, poids des graines et rendement}

Graines par silique et poids de 1000 graines des ramifications primaires:

Le tableau 6/A montre que dans les cages avec abeilles des deux essais, ce sont les siliques des plantes mâle-fertiles et des plantes mâle-stériles qui renferment plus de graines que celles des cages sans abeilles. On constate également que ce sont toujours les 
TABL. 4. - Hauteurs moyennes des tiges et longueurs moyennes

des ramifications primaires des plantes de colza mâle-fertile et mâle-stérile

dans les cages avec $(+A b)$ et sans $(-A b)$ abeilles et de plein champ $(T)$ : essai II, 1978.

TABL. 4. - Mean heights of the stems and mean lengths

of the primary ramifications of male-fertile and male-sterile rape-seed plants

in cages with (+ Hbees) and without ( - Hbees) honeybees

and in the field (T): Test II in 1978.

\begin{tabular}{|c|c|c|c|c|c|c|c|}
\hline & & $\begin{array}{l}+\mathrm{Ab} \\
+ \text { Hbees }\end{array}$ & $\begin{array}{l}-A b \\
- \text { Hbees }\end{array}$ & $\mathrm{T}$ & & $+\underset{/ T}{A b}$ & $\begin{array}{c}-\mathrm{Ab} \\
/ \mathrm{T}\end{array}$ \\
\hline \multirow{2}{*}{ H.T. } & $\begin{array}{l}\text { Mâle-fertile } \\
\text { Male-fertile }\end{array}$ & $\left(\begin{array}{c}169,37 \\
3,42) \\
(a)\end{array}\right.$ & $\left(\begin{array}{c}175,77 \\
1,93) \\
(b)\end{array}\right.$ & $\left(\begin{array}{c}160,03 \\
4,24) \\
(b)\end{array}\right.$ & $\mathrm{S}^{*}$ & 1,06 & 1,10 \\
\hline & $\begin{array}{l}\text { Mâle stérile } \\
\text { Male-sterile }\end{array}$ & $\left(\begin{array}{c}190,57 \\
5,26) \\
(a)\end{array}\right.$ & $\begin{array}{c}192,9 \\
( \pm \quad 2,46) \\
(b)(c)\end{array}$ & $\begin{array}{c}167,73 \\
\pm \quad 3,72) \\
(a)(c)\end{array}$ & $\mathrm{S}^{* * * *}$ & 1,14 & 1,15 \\
\hline \multicolumn{2}{|c|}{$\begin{array}{l}\text { Différences } \\
\text { Differences }\end{array}$} & $\mathrm{S}^{* * *}$ & $\mathrm{~S}^{* * *}$ & N.S. & & & \\
\hline \multirow{2}{*}{ L.R.P. } & $\begin{array}{l}\text { Mâle-fertile } \\
\text { Male-fertile }\end{array}$ & $\left(\begin{array}{c}58,67 \\
1,16) \\
(a)\end{array}\right.$ & $\left(\begin{array}{c}60,4 \\
\pm \quad 2,37) \\
(b)\end{array}\right.$ & $\begin{array}{c}50,67 \\
+\quad 2,46) \\
(a)(b)\end{array}$ & $\mathrm{S}^{* *}$ & 1,16 & 1,19 \\
\hline & $\begin{array}{l}\text { Mâle-stérile } \\
\text { Male-sterile }\end{array}$ & $\begin{array}{c}63,77 \\
( \pm 0,88) \\
(a)\end{array}$ & $\left(\begin{array}{c}72,57 \\
3,60) \\
(a)\end{array}\right.$ & $\left(\begin{array}{c}50,93 \\
2,80) \\
(a)\end{array}\right.$ & $\mathrm{S}^{* * * *}$ & 1,25 & 1,43 \\
\hline \multicolumn{2}{|c|}{$\begin{array}{l}\text { Différences } \\
\text { Differences }\end{array}$} & N.S. & $\mathrm{S}^{* * *}$ & N.S. & & & \\
\hline
\end{tabular}

Même légende que tableaux 1 et 2 .

H.T. : Hauteur des tiges

Height of stems.

L.R.P. : Longueur de la ramification primaire

Lengths of the primary ramifications.

siliques des plantes mâle-fertiles qui fournissent plus de graines que celles des plantes mâle-stériles, qu'elles soient en cages avec abeilles en cages sans abeilles ou en plein champ.

Le tableau 6/B ne fait pas apparaitre des différences importantes, sauf dans les cages sans abeilles de l'essai II.

\section{Production grainière $(R 1)$ de 1000 fleurs des ramifications primaires}

La production grainière de 1000 fleurs des plantes mâle-fertiles et mâle-stériles des cages avec abeilles est significativement plus élevée que celle des plantes mâlefertiles et mâle-stériles des cages sans abeilles (tableau 6/C). La production de graines de 1000 fleurs des plantes mâle-fertiles des cages avec abeilles des deux essais est en moyenne de 1,29 à 2,39 fois plus élevée qu'en cages sans abeilles, et de 1,05 à 1,1 fois plus qu'en plein champ. Chez les plantes mâle-stériles dans les cages avec abeilles, la variation est plus grande avec une production moyenne de graines de trois fois à 30 fois 
TABL. 5. - Nombres moyens de fleurs et nombres moyens de siliques de la ramification primaire des plantes de colza mâle-fertile et mâle-stérile dans les cages avec $(+A b)$ et sans $(-A b)$ abeilles et de plein champ (T): Essai II, 1978.

TABL. 5. - Mean numbers of flowers and mean numbers of siliquas of the primary ramifications of male-fertile and male-sterile rape seed plants in cages with (+Hbees) and without ( - Hbees) honeybees and in the field (T): Test II in 1978.

\begin{tabular}{|c|c|c|c|c|c|c|c|c|}
\hline & & $\begin{array}{l}+\mathrm{Ab} \\
+ \text { Hbees }\end{array}$ & $\begin{array}{l}-\mathrm{Ab} \\
- \text { Hbees }\end{array}$ & $\mathrm{T}$ & Diff. & $\begin{array}{l}+\mathrm{Ab} \\
1-\mathrm{Ab}\end{array}$ & $+\underset{T}{\mathrm{Ab}}$ & $\begin{array}{c}-\mathrm{Ab} \\
/ \mathrm{T}\end{array}$ \\
\hline \multirow{2}{*}{ N. Fl. } & $\begin{array}{l}\text { Mâle-fertile } \\
\text { Male-fertile }\end{array}$ & $\begin{array}{r}63,57 \\
+\quad 2,68)\end{array}$ & $\begin{array}{r}67,17 \\
(\quad 2,17)\end{array}$ & $\begin{array}{c}65,2 \\
(\quad 2,39)\end{array}$ & N.S. & 0,95 & 0,98 & 1,03 \\
\hline & $\begin{array}{l}\text { Mâle-stérile } \\
\text { Male-sterile }\end{array}$ & $\begin{array}{c}84,43 \\
\left(\begin{array}{c}2,63) \\
(a)\end{array}\right.\end{array}$ & $\begin{array}{c}87,7 \\
( \pm \quad 3,37) \\
(b)(c)\end{array}$ & $\begin{array}{c}75,03 \\
( \pm \quad 3,06) \\
(a)(c)\end{array}$ & $S^{*}$ & 0,96 & 1,13 & 1,17 \\
\hline \multicolumn{2}{|c|}{$\begin{array}{l}\text { Différences } \\
\text { Differences }\end{array}$} & $\mathrm{S}^{*}$ & $\mathrm{~S}^{*}$ & $\mathrm{~S}^{*}$ & & & & \\
\hline \multirow{3}{*}{ N. Si. } & $\begin{array}{l}\text { Mâle-fertile } \\
\text { Male-fertile }\end{array}$ & $\begin{array}{c}50,3 \\
+\quad 2,54)\end{array}$ & $\begin{array}{r}51,53 \\
( \pm \quad 2,55)\end{array}$ & 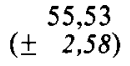 & N.S. & 0,98 & 0,91 & 0,93 \\
\hline & $\begin{array}{c}\text { Mâle-stérile } \\
\text { Male-sterile }\end{array}$ & $\begin{array}{c}65,07 \\
\left(\begin{array}{c}1,62) \\
(a)\end{array}\right.\end{array}$ & $\left(\begin{array}{c}41,27 \\
\pm \quad 3,27) \\
(a)\end{array}\right.$ & $\begin{array}{r}55,67 \\
\pm \quad 2,18 \\
(a)\end{array}$ & $S^{* * *}$ & 1,58 & 1,17 & 0,74 \\
\hline & $\begin{array}{l}\text { érences } \\
\text { erences }\end{array}$ & $S^{* * * *}$ & $\mathrm{~S}^{* * *}$ & N.S. & & & & \\
\hline
\end{tabular}

Même légende que tableaux 1 et 2 .

N. Fl. : Nombre de fleurs de la ramification primaire. Number of flowers on the primary ramification.

N. Si. : Nombre de siliques sur la ramification primaire. Number of siliquas on the primary ramification.

TABL. 6. - Moyennes des principales composantes du rendement des plantes de colza mâle-fertile et mâle-stérile dans les cages avec $(+A b)$ et sans ( $-A b)$ abeilles et de plein champ (T) : essais I, 1977 et II, 1978. Même légende que tableaux 1 et 2.

$\mathrm{G} / \mathrm{S}$ : nombre de graines par silique des ramifications primaires.

$P \quad$ : poids de 1000 graines (en centigrammes) des ramifications primaires.

R 1 : production de graines de 1000 fleurs au mètre carré (en quintaux à l'hectare) des ramifications primaires.

R 2 : rendement réel (en centigrammes) : poids total des graines récoltées sur toutes les plantes fertiles et stériles de chaque parcelle.

TABL. 6. - Averages of the main factors of yield of the male-fertile and male-sterile rape seed plants in cages with ( + Hbees) and without ( - Hbees) honeybees and in the field (T): Test I in 1977 and Test II in 1978. Legend as in tables 1 and 2.

$\mathrm{G} / \mathrm{S}$ : seed number per siliqua of the primary ramifications.

$P \quad$ : weight of 1000 seeds (in centigrammes) of the primary ramifications.

$\mathrm{R} 1$ : seed production of 1000 flowers per square metre (qx/ha) of the primary ramifications.

R 2 : actual yield (in centigrammes) : total weight of seeds harvested on all fertile and sterile plants of each plot. 


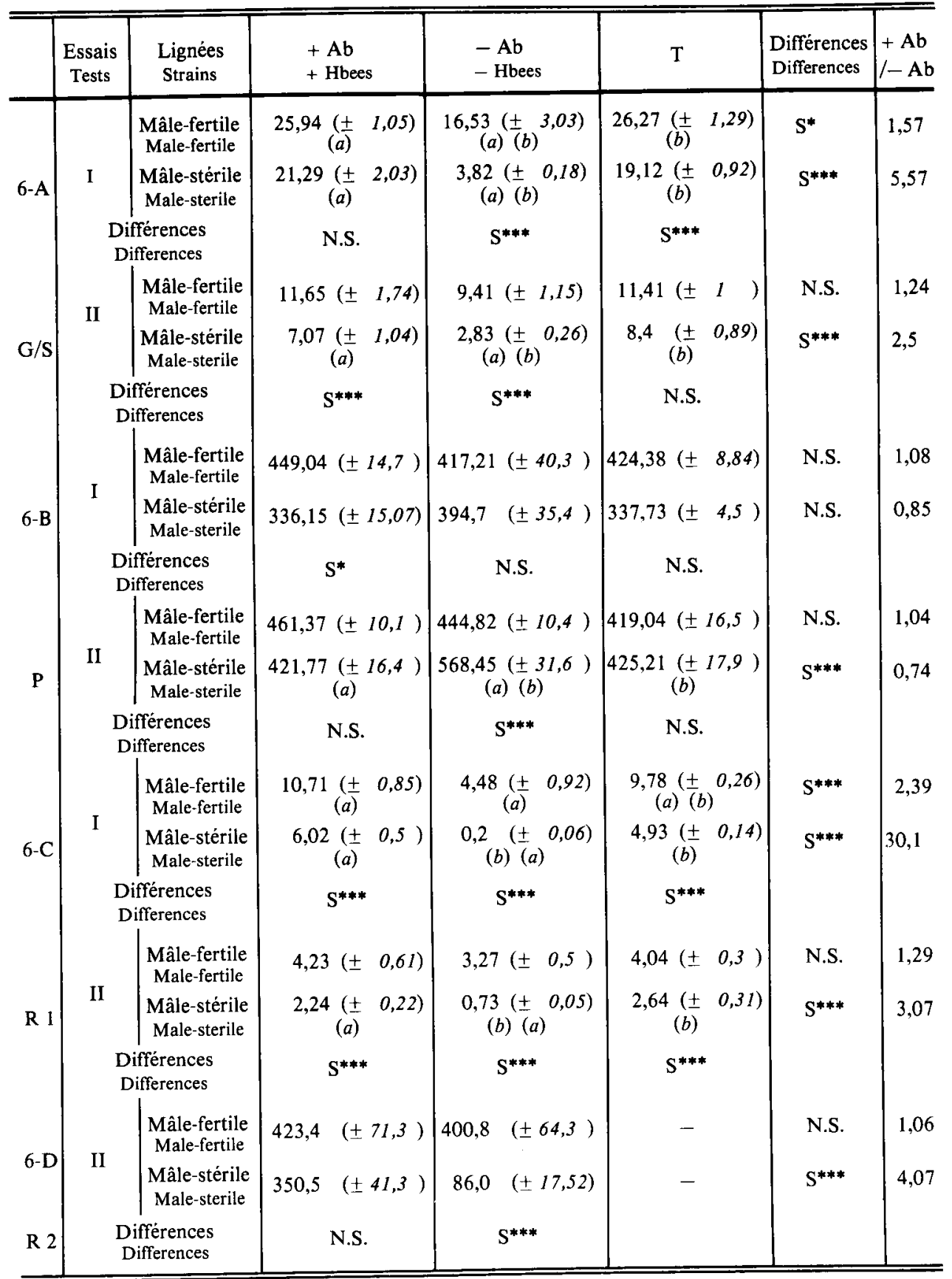


plus importante que celle des cages sans abeilles. Avec les plantes de plein champ, ce rapport est de 0,80 et 1,22 . Sous cages sans abeilles, la pollinisation par le vent ne représente finalement, chez les plantes mâle-stériles, que de $4 \%$ à $22 \%$ de la production grainière de 1000 fleurs des plantes mâle-fertiles non butinées. Rappelons que les cages diminuent notablement la vitesse du vent (de 60 à $90 \%$ ).

La production grainière de 1000 fleurs des plantes mâle-stériles représente $53 \%$ à $56 \%$ de la production des plantes mâle-fertiles en cage avec abeilles et $50 \%$ à $65 \%$ en plein champ.

\section{Rendement $(R 2)$ en graines des plantes entières}

Finalement, si l'on examine le rendement réel moyen des plantes mâle-fertiles des cages avec abeilles (tableau 6/D), on constate qu'il n'est pas significativement différent de celui des plantes mâle-fertiles des cages sans abeilles. On note seulement un léger gain de $6 \%$. Par contre, chez les plantes mâle-stériles dans les cages avec abeilles, le rendement est plus de quatre fois supérieur à celui des cages sans abeilles. Le rendement moyen dû au vent, chez les plantes mâle-stériles des cages sans abeilles n'est que les $21,4 \%$ de celui des plantes fertiles.

\section{DISCUSSION ET CONCLUSION}

Bien que nos études soient encore incomplètes, il est possible cependant de dire que, dans l'ensemble, les résultats obtenus permettent de confirmer l'influence de la pollinisation sur quelques caractères du colza. Elle s'est surtout manifestée sur les floraisons, les taux de nouaison et les composantes du rendement étudiées. Mais il est certain que ce sont surtout les plantes mâle-stériles qui ont permis de faire apparaitre significativement ces diverses influences dues à la pollinisation. Chez les plantes mâle-fertiles, elles ont été peu apparentes.

Des phénomènes de compensation portant sur la hauteur des tiges, sur le nombre de fleurs des ramifications primaires et sur la longueur des ramifications primaires entre parcelles situées sous cages avec abeilles et celles situées en extérieur interfèrent avec l'effet pollinisation. Ces effets sont d'ailleurs variables selon que la plante est mâlefertile ou mâle-stérile.

1. On trouve des quantités de fleurs plus élevées dans les cages sans abeilles que dans les cages avec abeilles quand les plantes de celles-ci et les plantes de plein champ amorcent justement leur défloraison. Ces résultats confirment d'ailleurs ceux de EwERT (1929) et ZANDER (1952).

Ces différences de floraison selon le mode de pollinisation peuvent s'expliquer, soit par la durée de vie plus longue des fleurs non butinées (BARBIER, 1978, MESQuida, non publié), soit par une production plus importante de fleurs sur la plante non visitée, soit 
aussi par la combinaison des deux phénomènes. La différence du nombre de fleurs résulterait d'une production de nouvelles fleurs aussi bien sur les ramifications primaires que secondaires ou tertiaires ou par une production tardive de nouvelles ramifications.

2. L'allongement des ramifications du colza, favorisé selon BARBIER par l'absence de butinage ou l'insuffisance de pollinisation, est confirmé, dans le cas des ramifications primaires des plantes mâle-stériles. Chez les plantes mâle-fertiles, le phénomène paraît masqué par un effet cage important.

3. L'influence favorable du butinage a été observé également sur les nouaisons, comme l'a montré BARBIER sur les ramifications primaires des plantes mâle-fertiles. Williams (1978) a d'ailleurs établi qu'en pratiquant une pollinisation manuelle, il était aussi possible d'augmenter les nouaisons.

4. L'influence du butinage sur l'augmentation de la production de siliques signalée par Meyerhoff (1954) a été observée sur les plantes mâle-stériles de nos essais. Chez celles-ci, le nombre de siliques sur les ramifications primaires butinées a été significativement plus élevé par le butinage, bien que l'on ait noté une plus faible production de fleurs. Les plantes mâle-fertiles n'ont pas permis de faire apparaitre cette influence.

5. Il n'a pas été possible de faire apparaitre de corrélations significatives entre les facteurs suivants : longueur de la ramification primaire et nombre de siliques; longueur de la ramification primaire et nombre de fleurs; longueur de la ramification primaire et taux de nouaison des plantes mâle-fertiles et mâle-stériles des cages avec abeilles, des cages sans abeilles et de plein champ.

6. Les fleurs butiriées donnent des siliques qui contiennent plus de graines sur les plantes mâle-fertiles, comme cela a déjà été établi par MEYERHOFF (1954), FORSTER et al. (1973) et BARBIER (1978). Ces graines, chez les plantes mâle-fertiles auraient tendance à être un peu plus grosses, comme l'écrit MEYerhofF; les différences n'étant cependant pas significatives. Elles sont par contre significativement plus petites chez les plantes mâle-stériles. Les plantes mâle-fertiles et les plantes mâle-stériles butinées ont, en tout cas, donné significativement une plus grosse production grainière de 1000 fleurs.

Chez les plantes mâle-fertiles, l'effet dû au butinage n'a que faiblement augmenté le rendement moyen total. Cette faible augmentation du rendement $(+6 \%)$ (non significative), liée au butinage, pourrait être néanmoins appréciable dans le cas du colza, dans la pratique agricole. De faibles accroissements du rendement, non significatifs, ont dejà été estimés non négligeables par de nombreux auteurs que nous avons précédemment cités (FuJTTA, 1939, ZANDER, 1952, etc.), sur des observations faites sous cages et en plein champ. 
Notons enfin que la pollinisation due au vent seul n'est pas négligeable puisqu'elle est responsable de taux de nouaisons assez élevés chez les plantes mâle-stériles. Mais son influence sur le rendement grainier des plantes mâle-stériles est par contre quatre fois plus faible que celle de l'abeille et du vent combinée dans nos conditions d'essais.

7. Il est aussi remarquable de constater que la très forte densité d'abeilles obtenue sur les plantes mâle-fertiles des cages où il y a eu à un moment donné près de 10 fois plus de butineuses qu'à l'extérieur, n'a pas permis d'améliorer significativement la pollinisation estimée par la mesure des nouaisons et des composantes du rendement. On peut émettre deux hypothèses : soit une efficacité pollinisatrice du vent, plus importante à l'extérieur que sous cage, ce qui masquerait l'effet densité d'abeilles, soit l'existence d'un seuil de densité d'abeilles largement dépassé, au point que même un excès de butinage ne permet plus d'amélioration de la production des plantes.

Cependant, dans ces comparaisons cages avec abeilles et cages sans abeilles, on sous-estime certainement l'efficacité pollinisatrice du vent en conditions de plein champ. On peut donc penser que les tendances observées quant à l'effet bénéfique du butinage sur les plantes mâle-fertiles pourraient très bien ne plus apparaitre sous l'effet du vent (transport du pollen ou secouement des plantes).

Notons enfin que cette étude nous a permis d'émettre un certain nombre d'hypothèses qu'il serait intéressant de vérifier, tant en cage qu'en plein champ : hypothèse concernant des phénomènes de compensation qui influent sur les rendements; hypothèse permettant de vérifier l'effet du butinage sur les caractères de la plante entière au niveau du nombre de fleurs produites sur toutes les ramifications, sur le nombre total de ramifications et sur leur taille respective, ainsi que sur le nombre total de siliques produites. Il serait aussi nécessaire de connaitre les potentialités de production grainière du colza, afin de définir le seuil de pollinisation qui permettrait de juger de l'intérêt que peut tirer la plante de la visite des fleurs par les abeilles et les autres pollinisateurs sauvages.

\section{REMERCIEMENTS}

Nous tenons à remercier tout particulièrement l'I.T.A.P.I. (1) et P.R.O.M.O.S.O.L. (2) pour l'aide que ces deux organismes nous ont apportée.

Reçu pour publication en mai 1981. Eingegangen im Mai 1981 .

(1) I.T.A.P.I. : Institut Technique de l'Apiculture. La Guyonnerie. Station de Recherches sur l'Abeille et les Insectes sociaux, 91440 Bures sur Yvette.

(2) P.R.O.M.O.S.O.L. : Association pour la Promotion de la Sélection des Semences Oléagineuses, 12, avenue George-V, 75008 Paris. 


\section{ZUSAMMENFASSUNG}

\section{DIE BEST ÄUBUNG VON MÄNNLICH-FERTILEM UND MÄNNLICH-STERILEM WINTERRAPS (BRASSICA NAPUS VAR. OLEIFERA) DURCH HONIGBIENEN. AUSWIRKUNGEN AUF DIE PHÄNOLOGIE UND DEN ERTRAG.}

In den Jahren 1977 und 1978 haben wir in Rennes (IIle-et-Vilaine) vergleichende Versuche in Käfigen mit und ohne Bienen und im Freiland mit zwei Sorten Winterraps durchgeführt : die männlich-fertile Sorte “ Primor ” und eine Sorte mit zytoplasmatischer männlicher Sterilität, um den Effekt der Bestäubung auf verschiedene phänologische Merkmale und auf die wichtigsten Komponenten des Ertrages zu überprüfen.

Es standen drei Gruppen mit 3 (1977) und 6 (1978) Wiederholungen zur Verfügung :

1. Parzellen im Käfig mit Bienen (ein Ableger pro Käfig mit zwei besetzten Dadant-Waben);

2. Parzellen im Käfig ohne Bienen;

3. Freilandparzellen ohne Käfig.

Die Käfige mit einem Volumen von $18 \mathrm{~m}^{3}(3 \times 3 \times 2 \mathrm{~m})$ waren mit einem Plastiknetz von $2 \mathrm{~mm}$ Maschenweite bedeckt. In jeder Parzelle befanden sich drei Reihen von $3 \mathrm{~m}$ Länge mit $0,5 \mathrm{~m}$ Intervall von männlich-fertilem Winterraps und drei Reihen von denselben Dimensionen von männlich-sterilem Winterraps.

Folgende Beobachtungen wurden angestellt:

- Sammelverhalten;

- Entwicklung der männlich-fertilen und der männlich-sterilen Blüten;

- folgende phänologische Merkmale : Rate der Samenbildung (in den ersten 50 Blüten der primären Verzweigungen); Gesamthöhe der Pflanzen; Länge und Anzahl der Blüten und der primären Verzweigungen;

- die wichtigsten Komponenten des Ertrages : Zahl der Schoten; Zahl der Samenkörner je Schote; Gewicht von 1000 Körnern; Samenproduktion von 1000 Blüten und Ertrag in Körnern.

Die phaenologischen Merkmale und die Ertragskomponenten wurden an primären Verzweigungen von je 5 Stengeln der männlich-fertilen und der männlich-sterilen Sorte bewertet, die zufällig aus jeder Parzelle ausgewertet wurde; nur der Samenertrag wurde aus sämtlichen Pflanzen ermittelt.

Folgende Resultate haben sich ergeben:

- die männlich-fertilen Pflanzen sind für die Bienen immer attraktiver als die männlich-sterilen, und die Sammelaktivität war in den Käfigen mit Bienen intensiver als im Freiland (Tab. 1).

Bei den männlich-sterilen Pflanzen zeigte sich der Einfluss der Bestäubung auf folgende Weise :

- Verringerung der Blütenzahl und der Blühdauer (Tab. 2);

- Vermehrung der Rate der Samenbildung (Tab. 3);

- Verringeruno der Grösse der primären Verzweigungen (Tab. 4);

- Verringerung der Blütenbildung (Tab. 5);

- Vermehrung der Zahl der Schoten (Tab. 5) und der Kornzahl pro Schote (Tab. 6 A) auf den primären Verzweigungen;

- Verringerung der Korngrösse (Tab. 6 B);

- Vermehrung der Samenproduktion je 1000 Blüten (Tab. 6 C); und des Ertrages (Tab. 6 D).

Bei der männlich-fertilen Rapssorte wurden dieselben Effekte des Befluges festgestellt, aber in viel geringerem Ausmass. Diese Effekte haben sich in signifikanter Weise vor allem bei der Rate des Samenansatzes gezeigt (Tab. 3) und bei der Samenproduktion je 1000 Blüten (Tab. 6 C). Der Samenertrag wurde nicht beeinflusst $(+6 \%$, ein nicht-signifikanter Unterschied; Tab. 6 D).

Der Effekt der reinen Windbestäubung auf den Samenansatz der männlich-sterilen Pflanzen in den bienenfreien Käfigen war in allen Fällen nicht zu vernachlässigen (Tab. 3), hatte aber schliesslich doch nur sehr geringen Einfluss auf die Erträge (Tab. 6 D). 


\section{SUMMARY \\ POLLINATION OF MALE-FERTILE AND MALE-STERILE WINTER RAPE-SEED \\ (BRASSICA NAPUS L. VAR. OLEIFERA METZGER) \\ BY THE HONEYBEE (APIS MELLIFICAL L.) \\ EFFECTS ON PHENOLOGY AND YIELD}

Materials and methods

In 1977 and 1978 in Rennes (Ille-et-Vilaine) we compared 2 strains of winter rape-seed in cages with and without honeybees and in the field : the male-fertile cultivar " Primor " and a cytoplasmic male-sterile strain, in order to show the effect of pollination on their various phenological features and their main yield factors.

We used 3 batches with 3 (1977) and 6 (1978) replicates:

1. Plots in cages with honeybees (a "Dadant" hive with 2 frames of bees per cage).

2. Plots in cages without honeybees.

3. Plots in the field without honeybees.

Cages, $18 \mathrm{~m}^{3}(3 \times 3 \times 2 \mathrm{~m})$, were covered with a plastic net of $2 \mathrm{~mm}$ mesh. Each plot comprised 3 lines, $3 \mathrm{~m}$ long and $0,50 \mathrm{~m}$ apart of male-fertile winter rape-seed and 3 lines of same size of male-sterile winter rape-seed.

Observations related to :

- the foraging behaviour;

- the evolution of male-fertile and male-sterile blossom-times;

- the following phenological features : fruit set rate (on the first 50 flowers of the primary ramifications); total height of plants; length and number of the flowers of the primary ramifications;

- the following main yield factors : number of siliquas; number of seeds per siliqua; weight of 1000 seeds; seed production of 1000 flowers and seed yield.

Phenological features and yield factors were estimated on the primary ramifications of 5 male-fertile and 5 male-sterile vines chosen at random in each plot, except the seed yield that related to all plants.

\section{Results}

Male-fertile plants were always more attractive to honeybees than male-sterile ones and foraging activity was more intense in cages with honeybees than in the field (Fig. 1). In male-sterile plants the influence of honeybee pollination found expression in :

- the decrease of the number of flowers and duration of blossom-times (Tabl. 2);

- the increase of fruit set rates (Tabl. 3);

- the decrease of the size of the primary ramifications (Tabl. 4);

- the decrease of the flower production (Tabl. 5).

- the increase of the number of siliquas (Tabl. 5) and the number of seeds per siliqua (Tabl. $6 \mathrm{~A}$ ) on the primary ramifications;

- the decrease of the seed size (Tabl. $6 \mathrm{~B}$ );

- the decrease of the seed production of 1000 flowers (Tabl. $6 \mathrm{C}$ ) and yield (Tabl. $6 \mathrm{D}$ ).

The same effects due to foraging were found in the male-fertile rape-seeds, but they were strongly reduced. They appeared especially significant on fruit set rates (Tabl. 3) and seed production of 1000 flowers (Tabl. $6 \mathrm{C})$. No influence was observed on the seed yield $(+6 \%$, not significant differences) (Tabl. $6 \mathrm{D})$.

In any case, the influence of wind pollination alone was slightly evident on fruit set of male-sterile plants of cages without bees (Tabl. 3), but the resultant yields were very low. 


\section{RÉFÉRENCES BIBLIOGRAPHIQUES}

Barbier E., 1977. - Pollinisation du colza. 26 Cong. Intern. d'Apic., 376. Apimondia, Bucarest.

Barbier E., 1978. - Pollinisation du colza par les abeilles. Rev. Fr. d'A pic., 365, 288-291.

BAUR R., 1943. - In Rives, 1957.

BeCKer Th., 1951. - Siebenjährige blütenbiologische Studien an den Cruziferen Brassica napus L., Brassica rapa L., Brassica oleracea L., Raphanus L. and Sinapis L. Z. Pflanzenzüchtg., 29, 222-240.

Belozerova E. I., 1960. - (Bees increase the seed crop from Winter rape). Pchelovodstvo, 37, 38-40. (En russe, résumé en anglais.)

Delaude A., TAsei J.-N., 1972. - Premières observations sur la pollinisation et la coulure des fleurs de luzerne en Charentes (Medicago sativa L.). Apidologie, 3, 79-97.

DEMIANOwiCz Z., 1965. - Recherche comparative concernant la production du nectar, la productivité du miel et de la récolte de semences prises sur 5 variétés de colza polonais d'automne. $20^{\prime}$ Cong. Intern. d'Apic., 321-324. Apimondia, Bucarest.

Downey R. K., Pawlowski S. H., Mc Ansh J., 1970. - In Mc Gregor. 1976.

EISIKowזch D., 1981. - Some aspects of pollination of oil-seed rape (Brassica napus L.). J. Agric. Sci. Camb., 96, 321-326.

EWERT R., 1929. - Die Befruchtung der Cruciferenblüte durch die Bienen. Arch. Bienenk., 10, $310-312$.

FeChNer E., 1927. - Untersuchungen über die Einwirkung eines Rückganges der Bienenzucht auf den Samenertrag einiger landwirtschaftlichen Kulturpflanzen. Arch. Bienenk., 8, 1-72.

Forster I. W., Clinch P. G., Palmer-Jones T., 1973. - Observations on the pollination of chou moellier (Brassica oleracea L.) N.Z.J. of Exp. Agric., 1, 46-48.

Free J. B., Nuttall P. H., 1968. - The pollination of oilseed rape (Brassica napus) and the behaviour of bees on the crop. J. Agric. Sci., Camb., 71, 91-94.

Fujit A M., 1939. - (Influence of honeybees on the fructification of rape). Bul. Imp. Zootechn. Exp. Stn. Chiba-Shi, 34, 1. (En japonais.)

HaRLE A., 1948. - Ist der Rapsglanzkäfer (Meligethes aeneus Fabr.) nur ein Schädling? Nachrichtenbl. dt. Pflanzenschutzdienstes, Berl., 2, 40-42.

HARLE A. 1951. - Zur Frage der Entragssteigerung bei Winterraps und Winterrübsen durch Einsartz von Honigbienen. Nachrichtenbl. dt. Pflanzenschutzdienstes (Braunschweig), 3, 20-22.

Jenkinson J. G., Glynne-Jones G. D., 1953. - Observations on the pollination of oil rape and broccoli. Bee World, 24, 173-177.

Joubert G., Tasei J.-N., Delaude A., 1977. - Pollinisation du trèfle violet diploïde (Trifolium pratense L.) par les Apoïdes et particulièrement par les abeilles domestiques hybrides (A pis mellifica caucasica $\times$ Apis mellifica ligustica). Apidologie, 8, 259-280.

Koutensky J., 1959. - (The pollinating effect of the Honey-Bee (Apis mellifera L.) on the Rise of hectare yields in Rape and White Mustard). Sb. Csl. Akad. Zemed. Ved., 5, 571-582. (En russe, résumé en anglais.)

Mac Gregor S. E., 1976. - Insect pollination of cultivated crop plants. Agriculture Handbook, 496, 1411 . U.S.D.A., Washington, D.C.

Mesquida J., Renard M., 1978. - Entomophilous pollination of male-sterile strains of Winter Rapeseed (Brassica napus L. Metzger var. oleifera) and a preliminary study of alternating devices. Proc. IVth Intern. Symp. on Pollination, 1, 49-57. Maryland.

Mesquida J., Renard M., 1978. - Entomophilous pollination of mâle-sterile strains of Winter Rapeseed stérile et les modalités de production de semences hybrides. Bull. Inf. Techn. C.E.T.I.O.M., 65, I-14.

MeyerhofF G., 1954. - Untersuchungen über die Wirkung des Bienenbefluges auf den Raps. $A r c h$. Geflügelz. Kleintierk., 3, 259-306.

MORICE J., 1960. - La sélection du colza d'hiver basée sur l'étude des composantes du rendement. $A n n$. Amélior. Plant., 1, 85-116. 
Morice J., 1976. - La contribution de la génétique et de la sélection végétale à l'amélioration de la production et de la qualité des produits des principaux oléagineux des régions tempérées. Rev. Fr. des corps gras, 23, 591-598.

Nicolaïsen W., 1943. - Züchtung von Raps. Z. Pflanzenzüchtg, 25, 362-379.

OLsson G., 1952. - "Investigations of the degree of cross-pollination in White mustard and rape ". Sverig. Utsädesfören. Tidskr., 62, 311-322. (in Swedish English Summary.)

OLssoN G., 1955. - Vindpollinering hos Korsblomstriga oljeväxter. Sver. Utsädesfören. Tidskr., 65, 418 422.

Persson Brita, 1956. - Korsbefruktningens betydelse och omfattning hos raps (Brassica napus L.) speciellt medavseende pä binas insats. Stat. Växtskyddsanst. Medd., 70, 1-36.

PraAgh Van J. P., Dustmann J. H., 1979. - Die Bestäubung Von Brassica napus var. oleifera im NordDeutschen Raum durch Honigbienen (Apis mellifera L.). Symp. Intern. Techn. Rationnelles de Pollinisation des cult., 60-66. Apimondia, Bucarest.

PriTsCh G., 1965. - Untersuchungen über die Steigerung der Oefruchterträge durch Honigbienen. Ved. Prace Vyzkum. Ustav. Vcelar. C.S.A.Z.V., 4, 157-163.

RHEIN W. Von, 1952. - Über die Duftlenkung der Bienen beim Raps in Jahre 1952 und ihre Ergebnisse. Hess. Biene, 88, 192-194.

Rives M., 1957. - Études sur la sélection du colza d'hiver. Ann. Amélior. Plant., 1, 61-107.

Rollier M., 1974. - Influence des facteurs climatiques sur le rendement du colza d'hiver. Proc. $4^{e}$ Intern. Rapskongress., 141-146. Giessen.

Rousselle P., 1981. - Étude de systèmes d'androstérilité chez le colza (Brassica napus L.). Thèse Univ. de Rennes, $109 \mathrm{p}$

SCHRIMPF D., 1954. - Untersuchungen über den Blüten und Schotenansatz bei Raps, Rübsen und Senf. $z$. Acker. Pflanzenbau, 97, 305-336.

Stolle G., 1954. - Ein Beitrag zur Ertragszüchtung beim Winterraps. Zuchter, 24, 202-215.

WhIte B., 1970. - Pollination of commercial rape seed crops. Australiasan Beekeepen, 72, 99-100.

WILLIAMS I. H., 1976. - The infestation of oilseed rape (Brassica napus $L$.) by the pests Meligethes aeneus Fab. and Ceuthorhynchus assimilis Payk. Univ. Lond. Ph. D. Thesis.

WILLIAMS I. H., 1978. - The pollination requirements of swede rape (Brassica napus L.) and of turnip rape (Brassica campestris L.). J. Agric. Sci., Camb., 91, 343-348.

Williams I. H., 1980. - Oil-Seed Rape and Beekeeping, particularly in Britain. Bee World, 4, 141-153. ZANDER E., 1952. -- Raps und Biene. Z. Bienenforsch., 1, 135-140. 